\title{
ON GENERALIZED VECTOR EQUILIBRIUM-LIKE PROBLEM IN TOPOLOGICAL VECTOR SPACES
}

\author{
L.-C. CENG, Y.-C. LIOU, C.-T. PANG, AND C.-F. WEN
}

Received 26 May, 2016

\begin{abstract}
In this paper, some existence results for generalized vector equilibrium-like problems (for short, GVELPs) are established by virtue of a generalized KKM-Fan theorem in topological vector spaces. In addition, some applications to vector optimization problems are also given. The results presented in this paper are the extension and improvement of the corresponding results of other authors.
\end{abstract}

2010 Mathematics Subject Classification: 49J40; 47H04; 47H05; 49J53

Keywords: KKM-Fan theorem, vector equilibrium-like problems, strong vector equilibrium-like problems, vector implicit equilibrium problems, vector optimization problems

\section{INTRODUCTION AND PRELIMINARIES}

The vector variational inequality (VVI) was first introduced in [1] in the setting of finite dimensional Euclidean space $\mathbf{R}^{\mathbf{n}}$. Subsequently, the VVI was generalized to infinite dimensional spaces in $[9,11,22,30]$. The vector variational-like inequality (VVLI), a generalization of VVI, was studied in [26,27] with applications in the vector optimization problem (VOP). The VVI and VVLI with set-valued mappings have been considered in $[16,18,31]$. In recent years some generalizations of VVI and VVLI have been further studied by many authors. For details, see e.g., [2-8, 13-15, 17, 19-21, 23-25, 28, 32, 34] and references therein. In 2008, Jabarootian and Zafarani [21] considered an extension of the VVLI with set-valued mapping, called generalized vector variational-like inequality (GVVLI). By using a generalized KKM-Fan theorem in [12], they established some results on the existence of solutions for this class of GVVLIs.

The first author was partially supported by the Innovation Program of Shanghai Municipal Education Commission (15ZZ068), Ph.D. Program Foundation of Ministry of Education of China (20123127110002), and Program for Outstanding Academic Leaders in Shanghai City (15XD1503100).

The second author was supported in part by MOST 103-2923-E-037-001-MY3, MOST 104-2221E-230-004 and Kaohsiung Medical University 'Aim for the Top Universities Grant, grant No. KMUTP103F00'. 
Next, we recall some concepts and notations. A nonempty subset $C$ of a vector space $Y$ is a convex cone if $\lambda C \subseteq C$ for all $\lambda \geq 0$ and $C+C \subseteq C$. A convex cone $C$ is pointed if $C \cap(-C)=\{0\}$. A cone $C$ is proper if it is properly contained in $Y$. Note that $C$ is a proper cone if and only if $0 \notin \operatorname{int} C$, where int $C$ denotes the interior of $C$. A pointed convex cone $C$ induces a partial order $\leq_{C}$ on $Y$ defined by $x \leq_{C} y$ whenever $y-x \in C$. In this case, $\left(Y, \leq_{C}\right)$ is an ordered vector space $\left(Y, \leq_{C}\right)$ with an order relation $\leq_{C}$. The weak order $\not_{\mathrm{int} C}$ on an ordered vector space $\left(Y, \leq_{C}\right)$ with $\operatorname{int} C \neq \varnothing$ is defined by $x \not_{\text {int } C} y$ whenever $y-x \notin \operatorname{int} C$.

Let $X$ and $Y$ be two topological spaces. We say that a multifunction $\varphi: X \rightarrow 2^{Y}$ is closed, or has closed graph if its graph was given by

$$
\mathcal{G}(\varphi)=\{(x, y) \in X \times Y: y \in \varphi(x)\}
$$

which is a closed subset of $X \times Y$.

For any two Hausdorff topological vector spaces (t.v.s.) $X$ and $Y$, let $\mathscr{L}(X, Y)$ denote the family of all continuous linear operators from $X$ into $Y$. When $Y$ is the set $\mathbf{R}$ of real numbers, $\mathscr{L}(X, Y)$ is the usual dual space $X^{*}$ of $X$. For any $x \in X$ and any $u \in \mathscr{L}(X, Y)$, we shall write the value $u(x)$ as $\langle u, x\rangle$. We suppose throughout this paper that $K$ is a nonempty closed convex subset of $X, T: K \rightarrow 2^{\mathscr{L}(X, Y)}$ is a setvalued mapping, $\Phi: \mathscr{L}(X, Y) \times K \times K \rightarrow Y, A: K \times \mathscr{L}(X, Y) \rightarrow \mathscr{L}(X, Y), \alpha: X \rightarrow$ $Y$ and $f: K \times K \rightarrow Y$ are four functions such that $f(x, x)=0$ for all $x \in X$, and $\{C(x): x \in K\}$ is a family of closed, convex and pointed cones of $Y$ (i.e., $C: K \rightarrow 2^{Y}$ is a cone mapping) such that int $C(x) \neq \varnothing$. for all $x \in K$.

Motivated and inspired by generalized vector equilibrium problems considered in Zeng and Yao [33,35], this work is to further extend the results of Jabarootian and Zafarani [21] to the setting of the generalized vector equilibrium-like problems (GVELPs). The main purpose of this article is the solvability of the following GVELP and its related problems in topological vector spaces.

Here, $\Phi(A(a, T(z)), x, y)=\{\Phi(A(a, u), x, y): u \in T(z)\}, \forall a, x, y, z \in K$.

Problem (I). Find $x_{0} \in K$ and $w_{0} \in A\left(x_{0}, T\left(x_{0}\right)\right)$ such that

$$
\Phi\left(w_{0}, x_{0}, y\right)+f\left(x_{0}, y\right) \not_{\mathrm{int} C}\left(x_{0}\right) 0, \quad \text { for all } y \in K .
$$

Problem (II). Find $x_{0} \in K$ such that for each $y \in K$

$$
\Phi\left(w, x_{0}, y\right)+f\left(x_{0}, y\right) \not_{\mathrm{int} C}\left(x_{0}\right) \alpha\left(y-x_{0}\right), \quad \text { for some } w \in A\left(x_{0}, T(y)\right) .
$$

Problem (III). Find $x_{0} \in K$ and $w_{0} \in A\left(x_{0}, T\left(x_{0}\right)\right)$ such that

$$
\Phi\left(w_{0}, x_{0}, y\right) \not \subset C\left(x_{0}\right) \backslash\{0\} 0, \quad \text { for all } y \in K .
$$

Problem (IV). Find $x_{0} \in K$ such that

$$
0 \leq C\left(x_{0}\right) \Phi\left(w, x_{0}, y\right), \quad \text { for all } y \in K \text { and } w \in A\left(x_{0}, T(y)\right) .
$$

Problem (V). Find $x_{0} \in K$ such that

$$
0 \leq C\left(x_{0}\right) \Phi\left(w, x_{0}, y\right), \quad \text { for all } y \in K \text { and } w \in A\left(x_{0}, T\left(x_{0}\right)\right) .
$$


In particular, if $A(x, u)=u$ for each $(x, u) \in K \times \mathscr{L}(X, Y)$ and $\Phi(w, x, y)=$ $\langle w, \eta(y, x)\rangle$ for each $(w, x, y) \in \mathscr{L}(X, Y) \times K \times K$, where $\eta: K \times K \rightarrow X$ is a function, then Problems (I)-(V) reduce to Problems (I)-(V) in [21], respectively. Problems (I) and (II) with $A(x, u)=u$ and $\Phi(w, x, y)=\langle w, \eta(y, x)\rangle$ become the simplified versions of the generalized vector variational-like inequality problems (GVVTIPs) in [24]. In this case, it is enough to replace $M(\cdot, T(\cdot)): K \rightarrow 2^{\mathscr{L}(X, Y)}$ in [24] with our mapping $T$. For Problem (II), by putting $\alpha \equiv 0$, we deduce in a similar way GVVTIP (II) in [24]. Problem (II) with $A(x, u)=u$ and $\Phi(w, x, y)=\langle w, \eta(y, x)\rangle$ is also a vector version of Problem (II) in [19].

Lemma 1 (see [9]). Let $\left(Y, \leq_{C}\right)$ be an ordered topological vector space with a closed, convex and pointed cone $C$ with int $C \neq \varnothing$. Then for each $x, y \in Y$, one has

(1) $y-x \in \operatorname{int} C$ and $y \notin \operatorname{int} C \Rightarrow x \notin \operatorname{int} C$.

(2) $y-x \in C$ and $y \notin$ int $C \Rightarrow x \notin$ int $C$.

(3) $y-x \in-\operatorname{int} C$ and $y \notin-\operatorname{int} C \Rightarrow x \notin-\operatorname{int} C$.

(4) $y-x \in-C$ and $y \notin-\operatorname{int} C \Rightarrow x \notin-\operatorname{int} C$.

We denote by $\mathcal{F}(X)$ the family of all nonempty finite subsets of $X$. Let $F: Y \rightarrow$ $2^{X}$ be a set-valued mapping. Then $F$ is said to be transfer closed-valued iff for each $(y, x) \in Y \times X$ with $x \notin F(y)$, there exists $y^{\prime} \in Y$ such that $x \notin \operatorname{cl} F\left(y^{\prime}\right)$. If $B \subseteq Y$ and $A \subseteq X$, then we call $F: B \rightarrow 2^{A}$ transfer closed-valued iff the multivalued mapping $y \mapsto F(y) \cap A$ is transfer closed-valued. When $X=Y$ and $A=B$, we call $F$ transfer closed-valued on $A$. Let $K$ be a convex subset of a vector space $X$. Then a mapping $F: K \rightarrow 2^{X}$ is called a KKM mapping iff for each nonempty finite subset $A$ of $K, \operatorname{conv} A \subset F(A)$, where conv $A$ denotes the convex hull of $A$, and $F(A)=\bigcup\{F(x): x \in A\}$.

Theorem 1 (see [12]). Let $K$ be a nonempty and convex subset of a Hausdorff t.v.s. $X$. Suppose that $\Gamma, \hat{\Gamma}: K \rightarrow 2^{K}$ are two set-valued mappings such that the following conditions are satisfied:

(A1) $\hat{\Gamma}(x) \subseteq \Gamma(x), \forall x \in K$;

(A2) $\hat{\Gamma}$ is a KKM map;

(A3) for each $A \in \mathcal{F}(K), \Gamma$ is transfer closed-valued on $\operatorname{conv} A$;

(A4) for each $A \in \mathcal{F}(K), \mathrm{cl}_{K}\left(\bigcap_{x \in \operatorname{conv} A} \Gamma(x)\right) \cap \operatorname{conv} A=\left(\bigcap_{x \in \operatorname{conv} A} \Gamma(x)\right) \cap$ $\operatorname{conv} A$;

(A5) there is a nonempty compact convex set $B \subseteq K$ such that $\operatorname{cl}_{K}\left(\bigcap_{x \in B} \Gamma(x)\right)$ is compact.

Then, $\bigcap_{x \in K} \Gamma(x) \neq \varnothing$.

Definition 1. Let $T: X \rightarrow 2^{Y}$ be a set-valued mapping. Then,

(i) $T$ is said to be upper semicontinuous (u.s.c.) iff, for each closed set $B \subset Y$,

$$
T^{-}(B)=\{x \in X: T(x) \cap B \neq \varnothing\} \text { is clised in } X ;
$$


(ii) $T$ is said to be lower semicontinuous (1.s.c.) iff, for each open set $B \subset Y$,

$$
T^{-}(B)=\{x \in X: T(x) \cap B \neq \varnothing\} \text { is open in } X ;
$$

(iii) $T$ is said to be closed (resp. open) iff, the set $G_{r}(T)=\{(x, y) \in X \times Y: y \in$ $T(x)\}$ is closed (resp. open) in $X \times Y$.

\section{GVELPS WITH MONOTONICITY}

Let $\mho$ be the family of all bounded subsets of $X$ whose union is total in $X$, i.e., the linear hull of $\bigcup\{S: S \in \mho\}$ is dense in $X$. Let $\mathcal{B}$ be a neighborhood base of 0 in $Y$. When $S$ runs through $\mho$ and $V$ through $\mathscr{B}$, the family

$$
M(S, V)=\{u \in \mathscr{L}(X, Y):\langle u, x\rangle \in V, \forall x \in S\}
$$

is a neighborhood base of 0 in $\mathscr{L}(X, Y)$ for a unique translation-invariant topology, called the topology of uniform convergence on the sets $S \in \mho$, or briefly the $\tau$ topology; see [29].

Definition 2. Let $\alpha: X \rightarrow Y$ with $\alpha(t z)=t^{p} \alpha(z) \forall t>0$ and $\forall z \in X$, where $p>1$. Then a function $\Phi: \mathscr{L}(X, Y) \times K \times K \rightarrow Y$ is said to be

(i) relaxed $\alpha-f$-pseudomonotone with respect to $T$ and $A$ if for each $x_{1}, x_{2}$, $y \in K$,

$$
\begin{aligned}
& \Phi\left(w_{1}, x_{1}, x_{2}\right)+f\left(x_{1}, x_{2}\right) \not_{\mathrm{int} C\left(x_{1}\right)} 0 \text { for all } w_{1} \in A\left(y, T x_{1}\right) \\
& \Rightarrow \Phi\left(w_{2}, x_{1}, x_{2}\right)+f\left(x_{1}, x_{2}\right) \not_{\mathrm{int} C}\left(x_{1}\right) \alpha\left(x_{2}-x_{1}\right) \text { for all } w_{2} \in A\left(y, T x_{2}\right) .
\end{aligned}
$$

(ii) relaxed $\alpha-f$-pseudomonotone-type with respect to $T$ and $A$ if for each $x_{1}, x_{2}$, $y \in K$,

$$
\begin{aligned}
& \Phi\left(w_{1}, x_{1}, x_{2}\right)+f\left(x_{1}, x_{2}\right) \not \not_{\mathrm{int} C}\left(x_{1}\right) 0 \text { for some } w_{1} \in A\left(y, T x_{1}\right) \\
& \Rightarrow \Phi\left(w_{2}, x_{1}, x_{2}\right)+f\left(x_{1}, x_{2}\right) \not_{\mathrm{int} C\left(x_{1}\right)} \alpha\left(x_{2}-x_{1}\right) \text { for some } w_{2} \in A\left(y, T x_{2}\right) .
\end{aligned}
$$

(iii) strongly pseudomonotone with respect to $T$ and $A$ if for each $x_{1}, x_{2}, y \in K$,

$$
\begin{gathered}
\Phi\left(w_{1}, x_{1}, x_{2}\right) \not_{C\left(x_{1}\right) \backslash\{0\}} 0 \text { for some } w_{1} \in A\left(y, T x_{1}\right) \\
\Rightarrow 0 \leq C\left(x_{1}\right) \Phi\left(w_{2}, x_{1}, x_{2}\right) \text { for all } w_{2} \in A\left(y, T x_{2}\right) .
\end{gathered}
$$

In particular, if $A(x, u)=u$ for each $(x, u) \in K \times \mathscr{L}(X, Y)$ and $\Phi(w, x, y)=$ $\langle w, \eta(y, x)\rangle$ for each $(w, x, y) \in \mathscr{L}(X, Y) \times K \times K$, where $\eta: K \times K \rightarrow X$ is a function, then the concepts in Definition 2 (i)-(iii) reduce to the ones in Definition 2 (i)-(iii) of [21], respectively.

Definition 3. A function $\Phi: \mathscr{L}(X, Y) \times K \times K \rightarrow Y$ is said to be upper hemicontinuous (u.h.c.) (resp. lower hemicontinuous (1.h.c.)) with respect to $T$ and $A$ if for each $x, y, z \in K$,

$$
t \in[0,1] \mapsto \Phi(A(z, T(x+t(y-x))), x, y)
$$

is u.s.c. (resp. 1.s.c.) at $0^{+}$. 
Definition 4. A set-valued mapping $F: K \rightarrow 2^{Y}$ is said to be $C$-convex where $C$ is a convex cone in $Y$ if for each $x, y \in K$ and $t \in[0,1]$ one has

$$
(1-t) F(x)+t F(y) \subseteq F((1-t) x+t y)+C .
$$

A single valued function $F: K \rightarrow Y$ is said to be $C$-convex if for each $x, y \in K$ and $t \in[0,1]$,

$$
F((1-t) x+t y) \leq_{C}(1-t) F(x)+t F(y) .
$$

Here, we consider a generalized vector version of Minty-type lemma.

Lemma 2. Let $C: K \rightarrow 2^{Y}$ be a cone mapping such that $\operatorname{int} C_{-} \neq \varnothing$, where $C_{-}=\bigcap_{x \in K} C(x)$. Assume that

(H1) for each $(w, x) \in \mathscr{L}(X, Y) \times K, \Phi(w, x, x)=0$ and $f$ is $C_{-}$-convex in the second variable;

(H2) $\Phi$ is continuous in the first variable and $C_{-}$-convex in the third variable, and $A$ is continuous in the second variable;

(H3) $\Phi$ is relaxed $\alpha-f$-pseudomonotone type and u.h.c. with respect to $T$ and $A$, where $T: K \rightarrow 2^{\mathscr{L}(X, Y)}$ takes compact values.

Then, Problems (I) and (II) are equivalent.

Proof. Problem (I) $\Rightarrow$ Problem (II) follows from the definition of relaxed $\alpha-f$ pseudomonotonicity type of $\Phi$ with respect to $T$ and $A$.

Conversely, suppose that we can find $x_{0} \in K$ such that for each $y \in K$

$$
\Phi\left(w, x_{0}, y\right)+f\left(x_{0}, y\right) \not_{\mathrm{int} C\left(x_{0}\right)} \alpha\left(y-x_{0}\right), \quad \text { for some } w \in A\left(x_{0}, T(y)\right) .
$$

This means that

$$
\Phi\left(A\left(x_{0}, T(y)\right), x_{0}, y\right)+f\left(x_{0}, y\right)-\alpha\left(y-x_{0}\right) \nsubseteq \underline{\operatorname{int} C}\left(x_{0}\right) .
$$

Assume to the contrary that, for any element $x_{0} \in K$, there exists $y_{0} \in K$ such that

$$
\Phi\left(w_{0}, x_{0}, y_{0}\right)+f\left(x_{0}, y_{0}\right) \leq_{\text {int } C\left(x_{0}\right)} 0, \quad \text { for all } w_{0} \in A\left(x_{0}, T\left(x_{0}\right)\right) .
$$

That is,

$$
\Phi\left(A\left(x_{0}, T\left(x_{0}\right)\right), x_{0}, y_{0}\right)+f\left(x_{0}, y_{0}\right) \subseteq-\operatorname{int} C\left(x_{0}\right) .
$$

Since $A$ is continuous in the second variable, by the compactness of $T\left(x_{0}\right)$ we know that $A\left(x_{0}, T\left(x_{0}\right)\right.$ is a compact set in $\mathscr{L}(X, Y)$. So, by the continuity of $\Phi$ in the first variable, we know that $\Phi\left(A\left(x_{0}, T\left(x_{0}\right)\right), x_{0}, y_{0}\right)+f\left(x_{0}, y_{0}\right)$ is a compact set in $Y$. Hence we can choose an open neighborhood $V$ of zero in $Y$ such that

$$
\Phi\left(A\left(x_{0}, T\left(x_{0}\right)\right), x_{0}, y_{0}\right)+f\left(x_{0}, y_{0}\right)+V \subseteq-\operatorname{int} C\left(x_{0}\right) .
$$

We consider $x_{t}=x_{0}+t\left(y_{0}-x_{0}\right)$ for $t \in[0,1]$. By the upper hemicontinuity of $\Phi$ with respect to $T$ and $A$, there exists $0<\beta<1$ such that, for $0<t<\beta$,

$$
\Phi\left(A\left(x_{0}, T\left(x_{t}\right)\right), x_{0}, y_{0}\right)+f\left(x_{0}, y_{0}\right)+V \subseteq-\operatorname{int} C\left(x_{0}\right) .
$$


Since $-C\left(x_{0}\right)$ is a cone, from condition (H1), we obtain

$$
\frac{1}{t} f\left(x_{0}, x_{t}\right)-f\left(x_{0}, y_{0}\right) \in-C\left(x_{0}\right) \text {. }
$$

Then, by (2.2) and (2.3), we deduce that

$$
\Phi\left(A\left(x_{0}, T\left(x_{t}\right)\right), x_{0}, y_{0}\right)+\frac{1}{t} f\left(x_{0}, x_{t}\right)+V \subseteq-\operatorname{int} C\left(x_{0}\right) .
$$

On the other hand, since $\Phi$ is $C_{-}$-convex in the third variable, we deduce that for all $v_{t} \in T\left(x_{t}\right)$,

$$
\frac{1}{t}\left[\Phi\left(A\left(x_{0}, v_{t}\right), x_{0}, x_{t}\right)-(1-t) \Phi\left(A\left(x_{0}, v_{t}\right), x_{0}, x_{0}\right)-t \Phi\left(A\left(x_{0}, v_{t}\right), x_{0}, y_{0}\right)\right] \in-C\left(x_{0}\right) .
$$

From condition (H1) and adding (2.4) and (2.5), for all $v_{t} \in T\left(x_{t}\right)$,

$$
\Phi\left(A\left(x_{0}, v_{t}\right), x_{0}, x_{t}\right)+f\left(x_{0}, x_{t}\right)+t V \subseteq-\operatorname{int} C\left(x_{0}\right) .
$$

As $\lim _{t \rightarrow 0^{+}} \frac{1}{t} \alpha\left(t\left(y_{0}-x_{0}\right)\right)=0$, for $t>0$ small enough, we have

$$
\Phi\left(A\left(x_{0}, v_{t}\right), x_{0}, x_{t}\right)+f\left(x_{0}, x_{t}\right)-\alpha\left(x_{t}-x_{0}\right) \subseteq-\operatorname{int} C\left(x_{0}\right) .
$$

But this contradicts (2.1).

Remark 1. Lemma 2 generalizes Lemma 2.1 of [25] to the GVELPs. It also improves and extends Theorem 3.1 of [24] and Lemma 2.5 of [19] because the VVLIs and GVVIs considered in [19,24] are replaced by the more general problems, i.e., GVELPs.

Theorem 2. Let $C: K \rightarrow 2^{Y}$ be a cone mapping such that $\operatorname{int} C_{-} \neq \varnothing$, where $C_{-}=\bigcap_{x \in K} C(x)$. Let hypotheses (H1)-(H3) of Lemma 2 hold and suppose that

(i) for each finite subset $D$ of $K$, the set-valued mapping $\Gamma: K \rightarrow 2^{K}$ defined by $\Gamma(y)=\left\{x \in K: \Phi(w, x, y)+f(x, y) \not_{\mathrm{int} C}(x) \alpha(y-x)\right.$, for some $\left.w \in A(x, T(y))\right\}$ is transfer closed valued on $\operatorname{conv}(D)$;

(ii) for each finite subset $D$ of $K, x, y \in \operatorname{conv}(D)$ and for each net $\left\{x_{\lambda}\right\}$ in $K$ converging to $x$, if $x_{\lambda} \in \Gamma(z)$ for all $z \in \operatorname{conv}(D)$ and for all $\lambda$, then $x \in \Gamma(y)$;

(iii) there exist a nonempty compact set $M \subset K$ and a nonempty compact convex set $B \subset K$ such that for each $x \in K \backslash M$, there exists $y \in B$ such that $x \notin \Gamma(y)$. Then, Problem (I) holds.

Proof. We define the set-valued mapping $\hat{\Gamma}: K \rightarrow 2^{K}$ by

$$
\hat{\Gamma}(y):=\left\{x \in K: \Phi(w, x, y)+f(x, y) \not_{\operatorname{int} C(x)} 0 \text {, for some } w \in A(x, T(x))\right\} .
$$

We show that $\hat{\Gamma}$ is a KKM mapping. Indeed, assume $\hat{\Gamma}$ is not a KKM mapping. Then there exists $\left\{x_{1}, x_{2}, \ldots, x_{n}\right\} \subset K, t_{i} \geq 0, i=1,2, \ldots, n$ with $\sum_{i=1}^{n} t_{i}=1$ such that $x=\sum_{i=1}^{n} t_{i} x_{i} \notin \bigcup_{i=1}^{n} \hat{\Gamma}\left(x_{i}\right)$. Thus for any $w \in A(x, T(x))$, we have

$$
\Phi\left(w, x, x_{i}\right)+f\left(x, x_{i}\right) \leq_{\operatorname{int} C(x)} 0, \quad i=1,2, \ldots, n ;
$$


therefore, we deduce that

$$
\sum_{i=1}^{n} t_{i} \Phi\left(w, x, x_{i}\right)+\sum_{i=1}^{n} t_{i} f\left(x, x_{i}\right) \leq \operatorname{int} C(x) 0 .
$$

On the other hand, since $f$ is $C_{-}$-convex in the second variable and $\Phi$ is $C_{-}$-convex in the third variable, we know from Eq. (2.6) that for each $w \in A(x, T(x))$

$$
\begin{aligned}
0 & =\Phi(w, x, x)+f(x, x) \\
& \leq C(x) \sum_{i=1}^{n} t_{i} \Phi\left(w, x, x_{i}\right)+\sum_{i=1}^{n} t_{i} f\left(x, x_{i}\right) \\
& \leq \text { int } C(x) 0,
\end{aligned}
$$

which leads to a contradiction that $C(x) \neq Y$. Hence, $\hat{\Gamma}$ is a KKM mapping. Since $\Phi$ is relaxed $\alpha$ - $f$-pseudomonotone-type with respect to $T$ and $A$, we have $\hat{\Gamma}(y) \subseteq \Gamma(y)$ for each $y \in K$. Hence, $\Gamma$ is also a KKM mapping. Thus all the conditions of Theorem 1 are fulfilled by the mappings $\hat{\Gamma}$ and $\Gamma$. Therefore, $\bigcap_{y \in K} \Gamma(y) \neq \varnothing$. Hence, Problem (II) holds and from Lemma 2, Problem (I) is deduced.

Remark 2. In the above theorem, if $\Gamma(y)$ is closed for all $y \in K$, then conditions (i) and (ii) hold. In the following results, we will establish the closedness of $\Gamma(y)$ for each $y \in K$.

Corollary 1. Let $X$ be a normed space and $(Y, \mathcal{T})$ be a t.v.s. Let $C: K \rightarrow 2^{Y}$ be a cone mapping such that int $C_{-} \neq \varnothing$, where $C_{-}=\bigcap_{x \in K} C(x)$. Let hypotheses (H1)-(H3) of Lemma 2 hold and suppose that

(i) the set-valued mapping $W: K \rightarrow 2^{Y}$ defined by $W(x)=Y \backslash(-\operatorname{int} C(x))$ has weakly closed graph;

(ii) $f$ is weakly continuous in the first variable and $\alpha$ is weakly continuous;

(iii) $A$ is continuous from $\sigma_{X} \times \tau$ to $\tau$, where $\sigma_{X}$ is the weak topology of $X$ and $\tau$ is the uniform convergence topology of $\mathscr{L}(X, Y)$;

(iv) for each $z \in K, \Phi(\cdot, \cdot, z): \mathscr{L}(X, Y) \times K \rightarrow Y$ is continuous from $\tau \times \sigma_{X}$ to $\sigma_{Y}$, where $\sigma_{Y}$ is the weak topology of $Y$;

(v) there exist a nonempty weakly compact set $M \subset K$ and a nonempty weakly compact convex set $B \subset K$ such that for each $x \in K \backslash M$, there exists $y \in B$ such that $x \notin \Gamma(y)$.

Then, Problem (I) holds.

Proof. By Theorem 2, it is enough to show that for each $y \in K, \Gamma(y)$ is weakly closed. Let $\left\{x_{\beta}\right\}$ be a net in $\Gamma(y)$ weakly convergent to $x_{0} \in K$. Since $x_{\beta} \in \Gamma(y)$, there exists $v_{\beta} \in \Gamma(y)$ satisfying

$$
\Phi\left(A\left(x_{\beta}, v_{\beta}\right), x_{\beta}, y\right)+f\left(x_{\beta}, y\right) \not_{\mathrm{int} C}\left(x_{\beta}\right) \alpha\left(y-x_{\beta}\right),
$$


that is,

$$
z_{\beta}=\Phi\left(A\left(x_{\beta}, v_{\beta}\right), x_{\beta}, y\right)+f\left(x_{\beta}, y\right)-\alpha\left(y-x_{\beta}\right) \notin-\operatorname{int} C\left(x_{\beta}\right) .
$$

Thus, $z_{\beta} \in W\left(x_{\beta}\right)$ and hence $\left(x_{\beta}, z_{\beta}\right) \in G_{r}(W)$. Since $T(y)$ is compact, $\left\{v_{\beta}\right\}$ has a convergent subnet in $T(y)$. Let $\left\{v_{\lambda}\right\}$ be a subnet of $\left\{v_{\beta}\right\}$ that converges to $v_{0} \in T(y)$. Since $A$ is continuous from $\sigma_{X} \times \tau$ to $\tau$, we know that $\left\{A\left(x_{\lambda}, v_{\lambda}\right)\right\}$ converges to $A\left(x_{0}, v_{0}\right) \in \mathscr{L}(X, Y)$. Also, since $\Phi(\cdot, \cdot, y): \mathscr{L}(X, Y) \times K \rightarrow Y$ is continuous from $\tau \times \sigma_{X}$ to $\sigma_{Y}$, we know that $\Phi\left(A\left(x_{\lambda}, v_{\lambda}\right), x_{\lambda}, y\right)$ converges weakly to $\Phi\left(A\left(x_{0}, v_{0}\right), x_{0}, y\right)$. Consequently, by the weak continuity of $f$ in the first variable and the weak continuity of $\alpha$ we deduce that $\left\{\Phi\left(A\left(x_{\lambda}, v_{\lambda}\right), x_{\lambda}, y\right)+f\left(x_{\lambda}, y\right)-\right.$ $\left.\alpha\left(y-x_{\lambda}\right)\right\}$ converges weakly to $z_{0}=\Phi\left(A\left(x_{0}, v_{0}\right), x_{0}, y\right)+f\left(x_{0}, y\right)-\alpha\left(y-x_{0}\right)$. Since $W$ has weakly closed graph and $\left(x_{\lambda}, z_{\lambda}\right) \in G_{r}(W)$ (due to (2.7)), we get $\left(x_{0}, z_{0}\right) \in G_{r}(W)$. Therefore,

$$
\Phi\left(A\left(x_{0}, v_{0}\right), x_{0}, y\right)+f\left(x_{0}, y\right)-\alpha\left(y-x_{0}\right) \notin-\operatorname{int} C\left(x_{0}\right) .
$$

That is, there exists $\tilde{w}\left(=A\left(x_{0}, v_{0}\right)\right) \in A\left(x_{0}, T(y)\right)$ such that

$$
\Phi\left(\tilde{w}, x_{0}, y\right)+f\left(x_{0}, y\right) \not_{\mathrm{int} C}\left(x_{0}\right) \alpha\left(y-x_{0}\right) .
$$

Thus, $x_{0} \in \Gamma(y)$, which completes the proof.

Remark 3. Corollary 1 generalizes Corollary 2.1 of [21] to the GVELPs. It also improves and extends Theorem 3.4 of [24] and Theorem 3.2 of [23] because the problems considered in our Corollary 1 are the more general problems, i.e., GVELPs.

Corollary 2. Let $X$ be metrizable. Let $C: K \rightarrow 2^{Y}$ be a cone mapping such that $\operatorname{int} C_{-} \neq \varnothing$, where $C_{-}=\bigcap_{x \in K} C(x)$. Let hypotheses (H1)-(H3) of Lemma 2 and condition (iii) of Theorem 2 hold, and suppose that

(i) the set-valued mapping $W: K \rightarrow 2^{Y}$ defined by $W(x)=Y \backslash(-\operatorname{int} C(x))$ is closed;

(ii) $f$ is continuous in the first variable and $\alpha$ is continuous;

(iii) for each $z \in K, \Phi(\cdot, \cdot, z): \mathscr{L}(X, Y) \times K \rightarrow Y$ is continuous and $A$ is continuous.

Then, Problem (I) holds.

Proof. By the similar proof to that of Corollary 1, we obtain the desired result.

Remark 4. It is easy to see that in the case where $K$ is a compact subset of $X$, we can omit the metrizability of $X$ in the above corollary. Moreover, Corollary 2 generalizes Corollary 2.2 of [21] to the GVELPs. It also improves and extends Theorems 2.1, 2.2 and 3.1 of [19], Theorem 3.1 of [32] and Theorem 3.4 of [23] because the problems considered in our Corollary 2 are the more general problems, i.e., GVELPs.

As another consequence of Theorem 2, when $\Phi$ is relaxed $\alpha$ - $f$-pseudomonotone with respect to $T$ and $A$, we also obtain a generalized version of Theorems 3.1-3.4 in [25] and hence Theorem 3.2 in [32]. 
Corollary 3. Assume $T$ has a continuous selection $g$ and let $C: K \rightarrow 2^{Y}$ be a cone mapping such that int $C_{-} \neq \varnothing$, where $C_{-}=\bigcap_{x \in K} C(x)$. Let hypotheses (H1)(H3) of Lemma 2 and conditions ( $i$ )-(iii) of Corollary 2 hold. If there exist a nonempty compact set $M \subset K$ and a nonempty compact convex set $B \subset K$ such that, for each $x \in K \backslash M$, there exists $y \in B$ such that

$$
\Phi(A(x, g(y)), x, y)+f(x, y) \leq_{\operatorname{int} C(x)} \alpha(y-x) .
$$

Then Problem (I) holds.

Proof. Since $\Phi$ is relaxed $\alpha$ - $f$-pseudomonotone with respect to $T$ and $A$, hence $\Phi$ is $\alpha$ - $f$-pseudomonotone-type with respect to $g$ (selection) and $A$. Thus, by Theorem 2 , it is enough to show that, for each $y \in K$, the set

$$
\Gamma(y)=\left\{x \in K: \Phi(w, x, y)+f(x, y) \not_{\mathrm{int} C(x)} \alpha(y-x) \quad \text { with } w=A(x, g(y))\right\}
$$

is closed. Indeed, let $\left\{x_{\beta}\right\}$ be a net in $\Gamma(y)$ convergent to $x_{0} \in K$. Since $x_{\beta} \in \Gamma(y)$,

$$
\Phi\left(w_{\beta}, x_{\beta}, y\right)+f\left(x_{\beta}, y\right) \not_{\mathrm{int} C\left(x_{\beta}\right)} \alpha\left(y-x_{\beta}\right) \quad \text { with } w_{\beta}=A\left(x_{\beta}, g(y)\right) ;
$$

that is,

$$
z_{\beta}=\Phi\left(A\left(x_{\beta}, g(y)\right), x_{\beta}, y\right)+f\left(x_{\beta}, y\right)-\alpha\left(y-x_{\beta}\right) \notin-\operatorname{int} C\left(x_{\beta}\right) .
$$

Thus, $z_{\beta} \in W\left(x_{\beta}\right)$ and hence $\left(x_{\beta}, z_{\beta}\right) \in G_{r}(W)$. By the continuity of $f$ in the first variable, continuity of $\alpha$ and $A$, and continuity of $\Phi(\cdot, \cdot, y)$, we have

$$
z_{0}=\lim _{\beta} z_{\beta}=\Phi\left(A\left(x_{0}, g(y)\right), x_{0}, y\right)+f\left(x_{0}, y\right)-\alpha\left(y-x_{0}\right) .
$$

Since $G_{r}(W)$ is closed, $\left(x_{0}, z_{0}\right) \in G_{r}(W)$ and hence,

$$
\Phi\left(A\left(x_{0}, g(y)\right), x_{0}, y\right)+f\left(x_{0}, y\right)-\alpha\left(y-x_{0}\right) \notin-\operatorname{int} C\left(x_{0}\right) ;
$$

that is,

$$
\Phi\left(\hat{w}, x_{0}, y\right)+f\left(x_{0}, y\right) \not_{\mathrm{int} C}\left(x_{0}\right) \alpha\left(y-x_{0}\right) \quad \text { with } \hat{w}=A\left(x_{0}, g(y)\right) .
$$

Therefore, $x_{0} \in \Gamma(y)$. This means that $\Gamma(y)$ is closed.

Corollary 3 generalizes Corollary 2.3 of [21] to the GVELPs. In order to obtain a solution of strong vector equilibrium-like problem, we need the following result. This result is a generalization of Lemma 2.2 in [21], and also a generalized vector version of Lemma 2.2 in [15].

Lemma 3. Let $C: K \rightarrow 2^{Y}$ be a cone mapping such that $\operatorname{int} C_{-} \neq \varnothing$, where $C_{-}=\bigcap_{x \in K} C(x)$. Let $\Phi$ be strongly pseudomonotone and l.h.c. with respect to $T$ and $A$. Assume that, for each $(w, x) \in \mathscr{L}(X, Y) \times K, \Phi(w, x, x)=0$ and $\Phi$ is affine in the third variable. Then Problems (III) and (IV) are equivalent. 
Proof. Problem (III) $\Rightarrow$ Problems (IV) follows from the definition of strong pseudomonotonicity of $\Phi$ with respect to $T$ and $A$.

Conversely, suppose that we can find $x_{0} \in K$ such that

$$
0 \leq C\left(x_{0}\right) \Phi\left(w, x_{0}, y\right), \quad \text { for all } y \in K \text { and } w \in A\left(x_{0}, T(y)\right) .
$$

We consider $x_{t}=x_{0}+t\left(y-x_{0}\right)$ for $t \in[0,1]$. Replacing $y$ by $x_{t}$ in Eq. (2.8), we have

$$
0 \leq C\left(x_{0}\right) \Phi\left(w, x_{0}, y_{t}\right), \quad \text { for all } w \in A\left(x_{0}, T\left(x_{t}\right)\right) .
$$

As $\Phi$ is affine in the third variable, $\Phi\left(w, x_{0}, x_{0}\right)=0, \forall w \in A\left(x_{0}, T\left(x_{t}\right)\right)$, and $C\left(x_{0}\right)$ is a cone, we deduce that for any $w \in A\left(x_{0}, T\left(x_{t}\right)\right)$

$$
\begin{aligned}
0 & \leq C\left(x_{0}\right) \Phi\left(w, x_{0}, y_{t}\right) \\
& =(1-t) \Phi\left(w, x_{0}, x_{0}\right)+t \Phi\left(w, x_{0}, y\right)=t \Phi\left(w, x_{0}, y\right) ;
\end{aligned}
$$

that is,

$$
\Phi\left(A\left(x_{0}, T\left(x_{t}\right)\right), x_{0}, y\right) \subseteq C\left(x_{0}\right) .
$$

Suppose on the contrary that

$$
\Phi\left(A\left(x_{0}, T\left(x_{0}\right)\right), x_{0}, y\right) \cap\left(Y \backslash C\left(x_{0}\right)\right) \neq \varnothing .
$$

As $\Phi$ is 1.h.c. with respect to $T$ and $A$, we have

$$
\Phi\left(A\left(x_{0}, T\left(x_{t}\right)\right), x_{0}, y\right) \cap\left(Y \backslash C\left(x_{0}\right)\right) \neq \varnothing,
$$

for sufficiently small $t$, which contradicts (2.9). Hence,

$$
\Phi\left(A\left(x_{0}, T\left(x_{0}\right)\right), x_{0}, y\right) \subseteq C\left(x_{0}\right), \quad \forall y \in K,
$$

which immediately yields

$$
\Phi\left(A\left(x_{0}, T\left(x_{0}\right)\right), x_{0}, y\right) \nsubseteq-C\left(x_{0}\right) \backslash\{0\}, \quad \forall y \in K .
$$

That is, there exists $w_{0} \in A\left(x_{0}, T\left(x_{0}\right)\right)$ such that

$$
\Phi\left(w_{0}, x_{0}, y\right) \not \subset C\left(x_{0}\right) \backslash\{0\} 0, \quad \text { for all } y \in K .
$$

This completes the proof.

Theorem 3. Let $C: K \rightarrow 2^{Y}$ be a cone mapping such that $\operatorname{int} C_{-} \neq \varnothing$, where $C_{-}=\bigcap_{x \in K} C(x)$. Let $\Phi$ be strongly pseudomonotone and l.h.c. with respect to $T$ and A. Suppose that

(i) for each finite subset $D$ of $K$, the set-valued mapping $\Gamma: K \rightarrow 2^{K}$ defined by

$$
\Gamma(y)=\left\{x \in K: 0 \leq_{C(x)} \Phi(w, x, y), \text { for all } w \in A(x, T(y))\right\}
$$

is transfer closed valued on $\operatorname{conv}(D)$;

(ii) for each finite subset $D$ of $K, x, y \in \operatorname{conv}(D)$ and for each net $\left\{x_{\lambda}\right\}$ in $K$ converging to $x$, if $x_{\lambda} \in \Gamma(z)$ for all $z \in \operatorname{conv}(D)$ and for all $\lambda$, then $x \in \Gamma(y)$;

(iii) for each $(w, x) \in \mathscr{L}(X, Y) \times K, \Phi(w, x, x)=0$ and $\Phi$ is affine in the third variable; 
(iv) there exist a nonempty compact set $M \subset K$ and a nonempty compact convex set $B \subset K$ such that for each $x \in K \backslash M$, there exists $y \in B$ such that $x \notin \Gamma(y)$.

Then, Problem (III) holds.

Proof. For each $y \in K$, we define the set-valued mapping

$$
\hat{\Gamma}(y):=\left\{x \in K: \Phi(w, x, y) \not_{C(x) \backslash\{0\}} 0, \quad \text { for some } w \in A(x, T(x))\right\} .
$$

We claim that $\hat{\Gamma}$ is a KKM mapping. Indeed, assume $\hat{\Gamma}$ is not a KKM mapping. Then there exist $\left\{x_{1}, x_{2}, \ldots, x_{n}\right\} \subset K, t_{i} \geq 0, i=1,2, \ldots, n$ with $\sum_{i=1}^{n} t_{i}=1$ such that $x=\sum_{i=1}^{n} t_{i} x_{i} \notin \bigcup_{i=1}^{n} \hat{\Gamma}\left(x_{i}\right)$. Thus for any $w \in A(x, T(x))$, we have

$$
\Phi\left(w, x, x_{i}\right) \leq_{C(x) \backslash\{0\}} 0, \quad i=1,2, \ldots, n,
$$

which yields $\sum_{i=1}^{n} t_{i} \Phi\left(w, x, x_{i}\right) \leq C(x) \backslash\{0\} 0$. Since for each $w \in A(x, T(x))$, $\Phi(w, x, x)=0$ and $\Phi$ is affine in the third variable, we obtain

$$
0=\Phi(w, x, x)=\sum_{i=1}^{n} t_{i} \Phi\left(w, x, x_{i}\right) \leq C(x) \backslash\{0\} 0,
$$

which is a contradiction. Hence, $\hat{\Gamma}$ is a KKM mapping. It is clear that strong pseudomonotonicity of $\Phi$ with respect to $T$ and $A$, guarantees that $\hat{\Gamma}(y) \subseteq \Gamma(y)$ for each $y \in K$. Thus all the conditions of Theorem 1 are fulfilled by the mappings $\hat{\Gamma}$ and $\Gamma$. Therefore, $\bigcap_{y \in K} \Gamma \neq \varnothing$. Consequently, Problem (IV) holds and from Lemma 3, Problem (III) is deduced.

Remark 5. Theorem 3 generalizes Theorem 2.2 of [21] to the GVELPs. In Theorem 3 , if the set $\Gamma(y)$ is closed for each $y \in K$, then conditions (i) and (ii) hold. In the following result, we will establish the closedness of $\Gamma(y)$ for each $y \in K$. This result generalizes Corollary 2.4 of [21] to the GVELPs. Moreover, it also extends and improves Theorem 2.3 in [15].

Corollary 4. Assume in Theorem 3, we replace conditions (i)-(ii) by the following conditions:

(i) the set-valued mapping $C: K \rightarrow 2^{Y}$ is closed;

(ii) for each $z \in K, \Phi(\cdot, \cdot, z): \mathscr{L}(X, Y) \times K \rightarrow Y$ is continuous and $A$ is continuous in the first variable.

Then, Problem (III) holds.

Proof. By Theorem 3, it is enough to show that for each $y \in K, \Gamma(y)$ is closed. Let $\left\{x_{\beta}\right\}$ be a net in $\Gamma(y)$ convergent to $x_{0} \in K$. Since $x_{\beta} \in \Gamma(y)$, for each $v \in T(y)$, we have $z_{\beta}=\Phi\left(A\left(x_{\beta}, v\right), x_{\beta}, y\right) \in C\left(x_{\beta}\right)$, which hence yields $\left(x_{\beta}, z_{\beta}\right) \in G_{r}(C)$. Also, since $\Phi(\cdot, \cdot, y): \mathscr{L}(X, Y) \times K \rightarrow Y$ is continuous and $A$ is continuous in the first variable, we know that the net $\left\{\Phi\left(A\left(x_{\beta}, v\right), x_{\beta}, y\right)\right\}$ converges to $\left(A\left(x_{0}, v\right), x_{0}, y\right)$; 
that is, $z_{0}=\lim _{\beta} z_{\beta}=\Phi\left(A\left(x_{0}, v\right), x_{0}, y\right)$. Since $G_{r}(C)$ is closed, we get $\left(x_{0}, z_{0}\right) \in$ $G_{r}(C)$ and hence, $\Phi\left(A\left(x_{0}, v\right), x_{0}, y\right) \in C\left(x_{0}\right)$ for all $v \in T(y)$; that is,

$$
0 \leq C\left(x_{0}\right) \Phi\left(w, x_{0}, y\right) \text {, for all } w \in A\left(x_{0}, T(y)\right) .
$$

This means that $x_{0} \in \Gamma(y)$. Therefore, $\Gamma(y)$ is closed.

\section{GVELPS WITHOUT MONOTONICITY}

In this section, some existence results for GVELPs without any monotonicity are obtained.

Theorem 4. Let $C: K \rightarrow 2^{Y}$ be a cone mapping such that $\operatorname{int} C_{-} \neq \varnothing$, where $C_{-}=\bigcap_{x \in K} C(x)$. Suppose that

(i) for each finite subset $D$ of $K$, the set-valued mapping $\Gamma: K \rightarrow 2^{K}$ defined by

$$
\Gamma(y)=\{x \in K: 0 \leq C(x) \Phi(w, x, y), \text { for all } w \in A(x, T(x))\}
$$

is transfer closed valued on $\operatorname{conv}(D)$;

(ii) for each finite subset $D$ of $K, x, y \in \operatorname{conv}(D)$ and for each net $\left\{x_{\lambda}\right\}$ in $K$ converging to $x$, if $x_{\lambda} \in \Gamma(z)$ for all $z \in \operatorname{conv}(D)$ and for all $\lambda$, then $x \in \Gamma(y)$;

(iii) for any fixed $x \in K$, the set $\left\{y \in K: 0 \not_{C(x)} \Phi(w, x, y)\right.$, for some $w \in$ $A(x, T(x))\}$ is convex;

(iv) For each $x \in K$, one has $0 \leq C(x) \Phi(w, x, x), \forall w \in A(x, T(x))$;

(v) there exist a nonempty compact set $M \subset K$ and a nonempty compact convex set $B \subset K$ such that for each $x \in K \backslash M$, there exists $y \in B$ such that $x \notin \Gamma(y)$.

Then, Problem (V) holds.

Proof. We claim that $\Gamma$ is a KKM mapping. Indeed, assume $\Gamma$ is not a KKM mapping. Then there exist $\left\{x_{1}, x_{2}, \ldots, x_{n}\right\} \subset K, t_{i} \geq 0, i=1,2, \ldots, n$ with $\sum_{i=1}^{n} t_{i}=$ 1 such that $x=\sum_{i=1}^{n} t_{i} x_{i} \notin \bigcup_{i=1}^{n} \Gamma\left(x_{i}\right)$. Thus, for any $i=1,2, \ldots, n$, we have

$$
\Phi\left(A(x, T(x)), x, x_{i}\right) \nsubseteq C(x) .
$$

Note that $\{y \in K: \Phi(A(x, T(x)), x, y) \not \subset C(x)\}=\{y \in K: 0 \not \subset C(x) \Phi(w, x, y)$, for some $w \in A(x, T(x))\}$. So, from condition (iii), it follows that $\Phi(A(x, T(x)), x, x) \nsubseteq$ $C(x)$, that is, there exists $w \in A(x, T(x))$ such that $0 \not \mathbb{Z}_{C(x)} \Phi(w, x, x)$, which contradicts condition (iv). Hence, $\Gamma$ is a KKM mapping. Thus all the conditions of Theorem 1 are fulfilled by the mappings $\hat{\Gamma}=\Gamma$. Therefore, $\bigcap_{y \in K} \Gamma(y) \neq \varnothing$. Consequently, Problem (V) holds.

Remark 6. In Theorem 4, we can replace conditions (iii)-(iv) by the following condition: (iii) ${ }^{\prime} \Phi$ is properly quasimonotone with respect to $T$ and $A$, i.e., for all $n \in \mathbf{N}$ and for all vectors $x_{1}, x_{2}, \ldots, x_{n} \in K$, and scalars $t_{i}>0, i=1,2, \ldots, n$ with $\sum_{i=1}^{n} t_{i}=1$ and $x=\sum_{i=1}^{n} t_{i} x_{i}, \Phi\left(A(x, T(x)), x, x_{i}\right) \subseteq C(x)$ holds for some $i$.

In fact, by a similar proof, we can show that $\Gamma$ is a KKM mapping. If $\Phi(w, x, y)=$ $\langle w, \eta(y, x)\rangle$ and $A(x, u)=u$ then Theorem 4 reduces to Theorem 3.1 of [21]. In this 
case, if we let $\eta(y, x)=y-g(x)$, then we conclude Theorem 2.1 in [14]. When $\Gamma(y)$ is closed for each $y \in Y$, then $\Gamma$ satisfies conditions (i) and (ii).

As a consequence of Theorem 4, in the following result we establish the closedness of $\Gamma$.

Corollary 5. Let $X$ be metrizable and suppose that

(i) for each $z \in K, \Phi(\cdot, \cdot, z): \mathscr{L}(X, Y) \times K \rightarrow Y$ is continuous and $A$ is continuous;

(ii) $T: K \rightarrow 2^{\mathscr{L}(X, Y)}$ is l.s.c.;

(iii) the set-valued mapping $W: K \rightarrow 2^{Y}$ defined by $W(x)=Y \backslash C(x)$ is open.

Then, Problem (V) holds.

Proof. By Theorem 4, it is enough to show that for each $y \in K, \Gamma(y)$ is closed. Indeed, let $\left\{x_{n}\right\}$ be a sequence in $\Gamma(y)$ convergent to $\bar{x} \in K$. By the lower semicontinuity of $T$, for each $v \in T(\bar{x})$ there exists a sequence $v_{n} \in T\left(x_{n}\right)$ converging to $v$. Set $z_{n}=\Phi\left(A\left(x_{n}, v_{n}\right), x_{n}, y\right)$. Then from $x_{n} \in \Gamma(y)$, we have $z_{n} \in C\left(x_{n}\right)$. Thus, $\left(x_{n}, z_{n}\right) \notin G_{r}(W)$. By the continuity of $A$, we know that $\left\{A\left(x_{n}, v_{n}\right)\right\}$ converges to $A(\bar{x}, v)$. Since $\Phi(\cdot, \cdot, y): \mathscr{L}(X, Y) \times K \rightarrow Y$ is continuous, it is clear that $\left\{\Phi\left(A\left(x_{n}, v_{n}\right), x_{n}, y\right)\right\}$ converges to $\left\{\Phi(A(\bar{x}, v), \bar{x}, y)\right.$; that is, $z=\lim _{n \rightarrow \infty} z_{n}=$ $\Phi(A(\bar{x}, v), \bar{x}, y)$. Since $G_{r}(W)$ is open, we have $(\bar{x}, z) \notin G_{r}(W)$, and hence $\bar{x} \in$ $\Gamma(y)$. This completes the proof.

Remark 7. If $\Phi(w, x, y)=\langle w, \eta(y, x)\rangle$ and $A(x, u)=u$ then Corollary 5 reduces to Corollary 3.1 of [21]. In this case, if we let $\eta(y, x)=y-g(x)$, then Corollary 5 establishes an existence theorem for strong vector implicit variational inequality problem in [20]. Thus we extend and improve Theorem 3.2 in [20] in many aspects. Corollary 5 also extends and improves Theorem 3.3 in [20] for the existence theorem of weak vector implicit variational inequality problem by using Theorem 3.1 (i) in [20]. As already mentioned, when $K$ is a compact subset of $X$, then we can omit the condition of metrizability of $X$.

We now establish an existence result for the GVELP without any monotonicity, which is a generalization of Theorem 3.2 in [21] for the strong vector variational-like inequality problem without any monotonicity.

Theorem 5. Let $C: K \rightarrow 2^{Y}$ be a cone mapping such that int $C_{-} \neq \varnothing$, where $C_{-}=\bigcap_{x \in K} C(x)$. Suppose that

(i) for each finite subset $D$ of $K$, the set-valued mapping $\Gamma: K \rightarrow 2^{K}$ defined by

$$
\Gamma(y)=\left\{x \in K: \Phi(w, x, y) \not_{C}(x) \backslash\{0\} 0 \text {, for some } w \in A(x, T(x))\right\}
$$

is transfer closed valued on $\operatorname{conv}(D)$;

(ii) for each finite subset $D$ of $K, x, y \in \operatorname{conv}(D)$ and for each net $\left\{x_{\lambda}\right\}$ in $K$ converging to $x$, if $x_{\lambda} \in \Gamma(z)$ for all $z \in \operatorname{conv}(D)$ and for all $\lambda$, then $x \in \Gamma(y)$;

(iii) for each $(w, x) \in \mathscr{L}(X, Y) \times K, \Phi(w, x, x)=0$ and $\Phi$ is affine in the third variable. ; 
(iv) there exist a nonempty compact set $M \subset K$ and a nonempty compact convex set $B \subset K$ such that for each $x \in K \backslash M$, there exists $y \in B$ such that $x \notin \Gamma(y)$. Then, Problem (III) holds.

Proof. By the similar proof to that of Theorem 3, on can show that $\Gamma$ is a KKM mapping. Thus all the conditions of Theorem 1 are fulfilled by the mapping $\hat{\Gamma}=$ $\Gamma$. Therefore, $\bigcap_{y \in K} \Gamma(y) \neq \varnothing$. Hence, there exists $x_{0} \in \bigcap_{y \in K} \Gamma(y)$ which is a solution for Problem (III).

As a consequence of Theorem 5, we obtain the following result which generalizes Theorem 3.2 of [21], and hence Theorem 2.1 of [15].

Corollary 6. Suppose that, in addition to conditions (iii) and (iv) of Theorem 5, for each $y \in K, \Gamma(y)$ is closed. Then, Problem (III) holds.

\section{Applications}

In this section, we consider solutions for the generalized weak optimization problem (VOP). Let $K$ be a convex subset of a Banach space $X, g: K \rightarrow \mathbf{R}^{\mathbf{n}}, g(x)=$ $\left(g_{1}(x), g_{2}(x), \ldots, g_{n}(x)\right)$ for $x \in K$, and $\{C(x): x \in K\}$ be a family of closed, convex and pointed cones of $\mathbf{R}^{\mathbf{n}}$ (i.e., $C: K \rightarrow 2^{\mathbf{R}^{\mathbf{n}}}$ is a cone mapping) such that $\mathbf{R}_{+}^{\mathbf{n}} \subseteq$ $C(x), \forall x \in K$. A point $y \in K$ is called a solution for the generalized weak VOP of $g$ if

$$
g(x) \not_{\mathrm{int} C(y)} g(y), \quad \text { for all } x \in K \text {. }
$$

Definition 5. Let $f: X \rightarrow \mathbf{R}$ be locally Lipschitz at a given point $x \in X$ and $v$ be any vector in $X$. The Clarke generalized directional derivative of $f$ at $x$ in the direction $v$, denoted by $f^{\circ}(x ; v)$, is defined by

$$
f^{\circ}(x ; v)=\limsup _{y \rightarrow x, t \downarrow 0} \frac{f(y+t v)-f(y)}{t} .
$$

Then, the Clarke generalized subdifferential of $f$ at $x$, denoted by $\partial^{c} f(x)$, is defined as

$$
\partial^{c} f(x)=\left\{\xi \in X^{*}: f^{\circ}(x ; v) \geq\langle\xi, v\rangle, \forall v \in X\right\} .
$$

Lemma 4 (see [10]). If $f: X \rightarrow \mathbf{R}$ is locally Lipschitz, then $\partial^{c} f(x)$ is a nonempty convex and weak ${ }^{*}$-compact valued. Moreover, $\partial^{c} f: X \rightarrow 2^{X^{*}}$ is norm-to-weak ${ }^{*}$ upper semicontinuous.

These definitions and properties can be extended to a locally Lipschitz vectorvalued function $g: X \rightarrow \mathbf{R}^{\mathbf{n}}$. Denote by $g_{i}, i=1,2, \ldots, n$ the components of $g$. The Clarke generalized subdifferential of $g$ at $x \in X$ is the set

$$
\partial^{c} g(x)=\partial^{c} g_{1}(x) \times \partial^{c} g_{2}(x) \times \cdots \times \partial^{c} g_{n}(x) .
$$


Throughout the remainder of this paper, let $\Phi: \mathscr{L}\left(X, \mathbf{R}^{\mathbf{n}}\right) \times K \times K \rightarrow \mathbf{R}^{\mathbf{n}}$ be a function and $g: K \rightarrow \mathbf{R}^{\mathbf{n}}$ be a function which has the Clarke generalized subdifferential on $K$.

Definition 6. The function $g$ is said to be invex with respect to $\Phi$ on $K$ if for each $x, y \in K$

$$
\Phi(u, x, y) \leq \mathbf{R}_{+}^{\mathbf{n}} g(y)-g(x), \quad \forall u \in \partial^{c} g(x) .
$$

Remark 8. Let $\eta: X \times X \rightarrow X$ be a function. Let the function $\Phi$ be defined as $\Phi(u, x, y)=\langle u, \eta(y, x)\rangle, \forall(u, x, y) \in \mathscr{L}\left(X, \mathbf{R}^{\mathbf{n}}\right) \times K \times K$. Assume that $g_{i}$ is locally Lipschitz on $K$ for $i=1,2, \ldots, n$. If

$$
\left\langle\zeta_{i}, \eta(y, x)\right\rangle \leq g_{i}(y)-g_{i}(x), \quad x, y \in K, \zeta_{i} \in \partial^{c} g_{i}(x), i=1,2, \ldots, n,
$$

then the concept of invex function of $g$ with respect to $\Phi$ on $K$, reduces to the one of invex function of $g$ with respect to $\eta$ on $K$ (see Definition 6 in [21]).

Definition 7. $\Phi$ is said to be $\partial^{c} g$-monotone if for each $x_{1}, x_{2} \in K$

$$
\Phi\left(u_{1}, x_{1}, x_{2}\right)+\Phi\left(u_{2}, x_{2}, x_{1}\right) \leq C\left(x_{1}\right) 0, \quad \forall u_{i} \in \partial^{c} g\left(x_{i}\right), i=1,2 .
$$

Definition 8. The function $\Phi: \mathscr{L}\left(X, \mathbf{R}^{\mathbf{n}}\right) \times K \times K \rightarrow \mathbf{R}^{\mathbf{n}}$ is called skew if $\Phi(u, x, y)$ $+\Phi(u, y, x)=0$ for each $(u, x, y) \in \mathscr{L}\left(X, \mathbf{R}^{\mathbf{n}}\right) \times K \times K$.

Lemma 5. Let $\Phi$ be skew and let $g$ be invex with respect to $\Phi$ on $K$. Then $\Phi$ is $\partial^{c} g$-monotone on $K$.

Proof. Suppose that $g$ is invex with respect to $\Phi$ on $K$. Then, for any $x, y \in K$, by the invexity of $g$, we obtain that

$$
\begin{gathered}
\Phi(u, x, y) \leq \mathbf{R}_{+}^{\mathbf{n}} g(y)-g(x), \forall u \in \partial^{c} g(x) \quad \text { and } \\
\Phi(v, y, x) \leq \mathbf{R}_{+}^{\mathbf{n}} g(x)-g(y), \forall v \in \partial^{c} g(y) .
\end{gathered}
$$

By adding these last two relations, we have

$$
\Phi(u, x, y)+\Phi(v, y, x) \leq \mathbf{R}_{+}^{\mathbf{n}} 0, \quad \text { for all } u \in \partial^{c} g(x), v \in \partial^{c} g(y) .
$$

Since $\mathbf{R}_{+}^{\mathbf{n}} \subseteq C(x)$, we deduce that for each $x, y \in K$

$$
\Phi(u, x, y)+\Phi(v, y, x) \leq C(x) 0, \quad \text { for all } u \in \partial^{c} g(x), v \in \partial^{c} g(y) .
$$

This means that $\Phi$ is $\partial^{c} g$-monotone on $K$.

Theorem 6. Let $g: K \rightarrow \mathbf{R}^{\mathbf{n}}$ be an invex function with respect to $\Phi$ on $K$. Given $x_{0} \in K$. We consider the following problems:

(P1) For each $y \in K$, there exists $u \in \partial^{c} g\left(x_{0}\right)$ such that $\Phi\left(u, x_{0}, y\right) \mathbb{Z}_{\mathrm{int} C}\left(x_{0}\right) 0$.

(P2) $x_{0}$ is a solution for the generalized weak VOP.

(P3) For each $y \in K$, there exists $v \in \partial^{c} g(y)$ such that $\Phi\left(v, x_{0}, y\right) \not_{\operatorname{int} C\left(x_{0}\right)} 0$.

Then, $(P 1) \Rightarrow(P 2)$. If $\Phi$ is skew, $(P 2) \Rightarrow(P 3)$. If $\Phi$ is affine in the third variable and $\Phi\left(\partial^{c} g(y), x, x\right)=\{0\}$ for each $x, y \in K,(P 3) \Rightarrow(P 1)$. 
Proof. (P1) $\Rightarrow(\mathrm{P} 2)$. Suppose that $x_{0}$ is not a solution for the generalized weak VOP. Then there exists $y \in K$ such that $g(y) \leq_{\operatorname{int} C\left(x_{0}\right)} g\left(x_{0}\right)$; that is,

$$
g\left(x_{0}\right)-g(y) \in \operatorname{int} C\left(x_{0}\right) \text {. }
$$

On the other hand, by the invexity of $g$, we have

$$
\Phi\left(u, x_{0}, y\right) \leq_{\mathbf{R}_{+}^{\mathrm{n}}} g(y)-g\left(x_{0}\right), \quad \forall u \in \partial^{c} g\left(x_{0}\right) ;
$$

that is, for all $u \in \partial^{c} g\left(x_{0}\right)$,

$$
\Phi\left(u, x_{0}, y\right) \in g(y)-g\left(x_{0}\right)-\mathbf{R}_{+}^{\mathbf{n}} \subseteq-\operatorname{int} C\left(x_{0}\right)-C\left(x_{0}\right) \subseteq-\operatorname{int} C\left(x_{0}\right) .
$$

Hence,

$$
\Phi\left(u, x_{0}, y\right) \leq_{\operatorname{int} C\left(x_{0}\right)} 0, \quad \forall u \in \partial^{c} g\left(x_{0}\right),
$$

which contradicts $(\mathrm{P} 1)$.

$(\mathrm{P} 2) \Rightarrow(\mathrm{P} 3)$. Suppose that $(\mathrm{P} 3)$ is not true. Then, for each $x_{0} \in K$, there exists $y_{0} \in K$ such that

$$
\Phi\left(v, x_{0}, y_{0}\right) \leq_{\operatorname{int} C\left(x_{0}\right)} 0, \quad \forall v \in \partial^{c} g\left(y_{0}\right) ;
$$

that is,

$$
\Phi\left(\partial^{c} g\left(y_{0}\right), x_{0}, y_{0}\right) \subseteq-\operatorname{int} C\left(x_{0}\right) .
$$

On the other hand, by the invexity of $g$, we have

$$
\Phi\left(u, y_{0}, x_{0}\right) \leq \mathbf{R}_{+}^{\mathbf{n}} g\left(x_{0}\right)-g\left(y_{0}\right), \quad \forall u \in \partial^{c} g\left(y_{0}\right) ;
$$

hence, since $\Phi$ is skew, we have

$$
-\Phi\left(u, x_{0}, y_{0}\right) \leq_{\mathbf{R}_{+}^{\mathbf{n}}} g\left(x_{0}\right)-g\left(y_{0}\right), \quad \forall u \in \partial^{c} g\left(y_{0}\right) ;
$$

therefore,

$$
g\left(x_{0}\right)-g\left(y_{0}\right) \in-\Phi\left(u, x_{0}, y_{0}\right)+\mathbf{R}_{+}^{\mathbf{n}} \subseteq \operatorname{int} C\left(x_{0}\right)+C\left(x_{0}\right) \subseteq \operatorname{int} C\left(x_{0}\right) .
$$

That is, $g\left(y_{0}\right) \leq_{\text {int } C\left(x_{0}\right)} g\left(x_{0}\right)$.

$(\mathrm{P} 3) \Rightarrow(\mathrm{P} 1)$. Since $g$ is invex with respect to $\Phi$ on $K$, by Lemma 5 we know that $\Phi$ is $\partial^{c} g$-monotone on $K$. Now if in Lemma 2, put $A(u, x)=u, f=0, \alpha=0$ and $T=\partial^{c} g$, then we obtain the desired result.

Theorem 6 generalizes Theorem 4.1 of [21] to the GVELPs. It also improves and extends the main result in [28]. As an application of Corollary 2, we establish an existence result for $(\mathrm{P} 2)$. This result is the extension and refinement of Theorem 3.1 in [21].

Theorem 7. Let $\Phi: \mathscr{L}(X, Y) \times K \times K \rightarrow \mathbf{R}^{\mathbf{n}}$ be a function and $g: K \rightarrow \mathbf{R}^{\mathbf{n}}$ be an invexity function with respect to $\Phi$ on $K$. Assume that the mapping $W: K \rightarrow$ $2^{\mathbf{R}^{\mathbf{n}}}$ defined by $W(x)=\mathbf{R}^{\mathbf{n}} \backslash(-\operatorname{int} C(x))$ is closed, $\Phi$ is skew and affine in the third variable, and for each $z \in K, \Phi(\cdot, \cdot, z): \mathscr{L}\left(X, \mathbf{R}^{\mathbf{n}}\right) \times K \rightarrow \mathbf{R}^{\mathbf{n}}$ is continuous. If there exist a nonempty compact set $M \subset K$ and a nonempty compact convex set $B \subset$ 
$K$ such that for each $x \in K \backslash M$, there exists $y \in B$ such that $\Phi\left(\partial^{c} g(x), x, y\right) \subseteq$ $-\operatorname{int} C(x)$. Then the generalized weak VOP has a solution.

Proof. Lemmas 4 and 5 imply that all the conditions of Corollary 2 are fulfilled. Hence, we deduce (P1) and then the proof follows from Theorem 6.

\section{REFERENCES}

[1] A. H. Ansari, A. H. Siddiqi, and J.-C. Yao, "Generalized vector variational-like inequalities and their scalarizations." in Vector variational inequalities and vector equilibria. Mathematical theories. Dordrecht: Kluwer Academic Publishers, 2000, pp. 17-37.

[2] Q. Ansari, A. Farajzadeh, and S. Schaible, "Existence of solutions of vector variational inequalities and vector complementarity problems." J. Glob. Optim., vol. 45, no. 2, pp. 297-307, 2009, doi: 10.1007/s10898-008-9375-X.

[3] L. Ceng, G. Chen, X. Huang, and J.-C. Yao, "Existence theorems for generalized vector variational inequalities with pseudomonotonicity and their applications." Taiwanese J. Math., vol. 12, no. 1, pp. 151-172, 2008.

[4] L. Ceng, P. Cubiotti, and J. Yao, "Existence of vector mixed variational inequalities in Banach spaces." Nonlinear Anal., Theory Methods Appl., Ser. A, Theory Methods, vol. 70, no. 3, pp. 1239-1256, 2009, doi: 10.1016/j.na.2008.01.039.

[5] L. Ceng, G. Mastroeni, and J. Yao, "Existence of solutions and variational principles for generalized vector systems." J. Optim. Theory Appl., vol. 137, no. 3, pp. 485-495, 2008, doi: 10.1007/s10957-007-9348-0.

[6] L. Ceng, S. Schaible, and J. Yao, "Existence of solutions for generalized vector variational-like inequalities." J. Optim. Theory Appl., vol. 137, no. 1, pp. 121-133, 2008, doi: 10.1007/s10957007-9336-4.

[7] L.-C. Ceng and S. Huang, "Existence theorems for generalized vector variational inequalities with a variable ordering relation." J. Glob. Optim., vol. 46, no. 4, pp. 521-535, 2010, doi: 10.1007/s10898-009-9436-9.

[8] L.-C. Ceng and S. Kum, "On generalized vector implicit variational inequalities and complementarity problems," Taiwanese Journal of Mathematics, vol. 11, no. 3, pp. 621-636, 2007.

[9] G. Chen, "Existence of solutions for a vector variational inequality: An extension of the Hartmann-Stampacchia theorem.” J. Optim. Theory Appl., vol. 74, no. 3, pp. 445-456, 1992, doi: 10.1007/BF00940320.

[10] F. Clarke, Y. Ledyaev, R. Stern, and P. Wolenski, Nonsmooth Analysis and Control Theory. Springer, 1998. doi: 10.1007/b97650.

[11] A. Daniilidis and N. Hadjisavvas, "Existence theorems for vector variational inequalities." Bull. Aust. Math. Soc., vol. 54, no. 3, pp. 473-481, 1996, doi: 10.1017/S0004972700021882.

[12] M. Fakhar and J. Zafarani, "Generalized vector equilibrium problems for pseudomonotone multivalued bifunctions." J. Optim. Theory Appl., vol. 126, no. 1, pp. 109-124, 2005, doi: 10.1007/s10957-005-2663-4.

[13] N. Fang, Y.P.and Huang, "Variational-like inequalities with generalized monotone mappings in banach spaces," Journal of Optimization Theory and Applications, vol. 118, no. 2, pp. 327-338, 2003, doi: 10.1023/A:1025499305742.

[14] Y.-P. Fang and N.-J. Huang, "Existence results for systems of strong implicit vector variational inequalities." Acta Math. Hung., vol. 103, no. 4, pp. 265-279, 2004, doi: 10.1023/B:AMHU.0000028828.52601.9e.

[15] Y.-P. Fang and N.-J. Huang, "Strong vector variational inequalities in Banach spaces." Appl. Math. Lett., vol. 19, no. 4, pp. 362-368, 2006, doi: 10.1016/j.aml.2005.06.008. 
[16] F. Giannessi, "Theorems of alternative, quadratic programs and complementarity problems." Variational inequalities and complementarity problems, theory and applications, Proc. int. School Math., Erice/Sicily 1978, 151-186 (1980)., 1980.

[17] F. Giannessi and A. Maugeri, Variational Analysis and Applications. Nonconvex Optimization and Its Applications. Springer, 2005. doi: 10.1007/b105059.

[18] N. Hadjisavvas and S. Schaible, "From scalar to vector equilibrium problems in the quasimonotone case.” J. Optim. Theory Appl., vol. 96, no. 2, pp. 297-309, 1998, doi: 10.1023/A:1022666014055.

[19] N.-J. Huang and Y.-P. Fang, "On vector variational inequalities in reflexive Banach spaces." $J$. Glob. Optim., vol. 32, no. 4, pp. 495-505, 2005, doi: 10.1007/s10898-003-2686-z.

[20] N.-J. Huang and J. Li, "On vector implicit variational inequalities and complementarity problems." J. Glob. Optim., vol. 34, no. 3, pp. 399-408, 2006, doi: 10.1007/s10898-004-1938-x.

[21] T. Jabarootian and J. Zafarani, "Generalized vector variational-like inequalities," Journal of Optimization Theory and Applications, vol. 136, no. 1, pp. 15-30, 2008, doi: 10.1007/s10957-0079300-3.

[22] I. Konnov and J. Yao, “On the generalized vector variational inequality problem.” J. Math. Anal. Appl., vol. 206, no. 1, pp. 42-58, art. no. ay975 192, 1997, doi: 10.1006/jmaa.1997.5192.

[23] S. Kum and W. K. Kim, "Generalized vector variational and quasi-variational inequalities with operator solutions," Journal of Global Optimization, vol. 32, no. 4, pp. 581-595, 2005, doi: 10.1007/s10898-004-2695-6.

[24] B.-S. Lee, S.-S. Chang, J. S. Jung, and S.-J. Lee, "Generalized vector version of Minty's lemma and applications." Comput. Math. Appl., vol. 45, no. 4-5, pp. 647-653, 2003, doi: 10.1016/S08981221(03)00024-5.

[25] J. Li and Z.-Q. He, "Gap functions and existence of solutions to generalized vector variational inequalities." Appl. Math. Lett., vol. 18, no. 9, pp. 989-1000, 2005, doi: 10.1016/j.aml.2004.06.029.

[26] K. Lin, D. Yang, and J. Yao, "Generalized vector variational inequalities." J. Optim. Theory Appl., vol. 92, no. 1, pp. 117-125, 1997, doi: 10.1023/A:1022640130410.

[27] L.-J. Lin, "Pre-vector variational inequalities." Bull. Aust. Math. Soc., vol. 53, no. 1, pp. 63-70, 1996, doi: 10.1017/S0004972700016725.

[28] S. Mishra and S. Wang, "Vector variational-like inequalities and non-smooth vector optimization problems," Nonlinear Analysis: Theory, Methods \& Applications, vol. 64, no. 9, pp. 1939 - 1945, 2006, doi: http://dx.doi.org/10.1016/j.na.2005.07.030.

[29] H. Schaefer, Topological Vector Spaces. Springer, 1999. doi: 10.1007/978-1-4612-1468-7.

[30] X. Yang, "Vector variational inequality and vector pseudolinear optimization." J. Optim. Theory Appl., vol. 95, no. 3, pp. 729-734, 1997, doi: 10.1023/A:1022694427027.

[31] X. Yang, "Vector variational inequality and vector pseudolinear optimization." J. Optim. Theory Appl., vol. 95, no. 3, pp. 729-734, 1997, doi: 10.1023/A:1022694427027.

[32] X. Yang and J. Yao, "Gap functions and existence of solutions to set-valued vector variational inequalities.” J. Optim. Theory Appl., vol. 115, no. 2, pp. 407-417, 2002, doi: 10.1023/A:1020844423345.

[33] L.-C. Zeng and J.-C. Yao, "An existence result for generalized vector equilibrium problems without pseudomonotonicity.” Appl. Math. Lett., vol. 19, no. 12, pp. 1320-1326, 2006, doi: 10.1016/j.aml.2005.09.010.

[34] L.-C. Zeng and J.-C. Yao, "Existence of solutions of generalized vector variational inequalities in reflexive Banach spaces." J. Glob. Optim., vol. 36, no. 4, pp. 483-497, 2006, doi: 10.1007/s10898005-5509-6.

[35] L.-C. Zeng and J.-C. Yao, “Generalized Minty's Lemma for generalized vector equilibrium problems.” Appl. Math. Lett., vol. 20, no. 1, pp. 32-37, 2007, doi: 10.1016/j.aml.2006.02.019. 
Authors' addresses

\section{L.-C. Ceng}

Department of Mathematics, Shanghai Normal University, Shanghai, 200234, China

E-mail address: zenglc@hotmail.com

Y.-C. Liou

Department of Healthcare Administration and Medical Informatics, Kaohsiung Medical University, Kaohsiung, 80708, Taiwan

E-mail address: simplex_liou@hotmail.com

\section{C.-T. Pang}

Department of Information Management, Yuan Ze University, Chung-Li, 32003, Taiwan

E-mail address: imctpangesaturn.yzu.edu.tw

\section{C.-F. Wen}

Center for Fundamental Science, and Center for Nonlinear Analysis and Optimization, Kaohsiung Medical University, Kaohsiung, 80708, Taiwan

E-mail address: cfwen@kmu.edu.tw 